\title{
Findings of Patterns in Pre-historic Architecture- Case Study of The Pyramid of CHEOPS
}

\author{
Fouad A. Ghoussayni*1, Amer Ali ${ }^{2}$, Ali Bayyati ${ }^{3}$ \\ ${ }^{1}$ London South Bank University, School of the Built Environment and Architecture \\ London, United Kingdom \\ ${ }^{2}$ London South Bank University, School of the Built Environment and Architecture \\ London, United Kingdom \\ ${ }^{3}$ London South Bank University, School of the Built Environment and Architecture \\ London, United Kingdom \\ Corresponding author: ghoussaf@lsbu.ac.uk
}

\begin{abstract}
Ghoussayni, A. Fouad. Ali, Amer and Bayyati, Ali. 2018. Findings of patterns in pre-historic architecture: case study of the pyramid of Cheops. Lebanese Science Journal, 19(2): 247-257.

There has been an increasing need to explain the geometric representation of the Pyramid of Cheops in Egypt. Some divergent approaches have been attempted, which need further exploration. The aim of this research paper is to unfold the mysteries behind one of the most notable notions of our universe, as it lies hidden in the dark configuration of space, the void. The space which incubates patterns depicted in the form of a pyramid can also nurse buried void. The approach used in this investigation is based on mathematical illustrations, which led to conclude that void versus matter is a constant strain for balance, and has been ever since the first formation of the apex of a pyramid. The main findings of this paper are that the manifest states, as shown in the schematic diagrams depicting the shape of the pyramid of Cheops, are in the form of balance between void and matter. The key conclusion is that there is a link between the graphical representation of the distributed building blocks in any horizontal layer of the pyramid of Cheops, and the general depiction of its form and angle derived from a geometric balance in the arrangement of its building blocks.
\end{abstract}

Keywords: Cheops, pyramid, geometry, historic architecture, void-matter balance, symmetry. 


\section{INTRODUCTION}

Although there have been various attempts to explain the geometric representation of the pyramid of Cheops in Egypt, there is a need for further exploration of the topic. This research paper aims to unfold the mysteries behind one of the most famous architectural landmarks of our world, in context of the matter it comprises as well as its hidden depths in the dark configuration of space, the void.

"The term "true" pyramid is used to differentiate the true ancient Egyptian pyramids from other more common step pyramids, found all over the world. The fact that the Egyptian pyramids are the only true pyramids in the world culminating in an Apex point has two implications: The first is the need for extreme precision on a grand scale. To aim for a point $146.6 \mathrm{~m}$ (280 royal cubits (Rc)) up in the sky with only a plumb line and a string, implies the crucial need for numerous points of measure (Bryn, 2010).

Historically, the Egyptian pyramids were regarded as piles of stone that needed to be moved one on top of the other (Bryn, 2010). However, this assumption of any kind of piling has been proved to be erroneous. The geometric principle at work in the Cheops pyramid is the arrangement of blocks of stone on a horizontal plane according to a particular geometric organization.

The masonry of these step pyramids was clearly too roughly constructed to act as a reference for the outer slope (Lehner, 1997). The findings below are of extreme significance in explaining the relation between the horizontal arrangements and the slope of the pyramid of Cheops.

\section{Expanding the proof}

Void versus matter is in constant balance and progression ever since the first apex of a pyramid with a square base was formed. When the scale changes, so does the level of complexity changes; the simpler the scale, the greater the sophistication. Conversely, the more complex the scale, the simpler the level of sophistication, making it evident that scale and sophistication work differently.

The different configurations of pixels and voids is in accordance with the balance of states that will provide an insight into the relationship between the horizontal arrangement of each level of stone with respect to the slope of the surface of the pyramids of Cheops.

Matter and embossed configurations of void expand at an exponential rate. However, ever since the first dialogue and formation, matter accelerates in a defined path of evolution that casts behind it an accumulation of establishments that rely on a 
single rule: the perpetual relationship between matter and void in continual balance, which brings structures into existence.

It is as though the apex is declaring the rise of the earliest progression right from the start, from a simple point to the endless journey across the universe. The scale and precision are prospectively the title of this human-made structure.

The questions that people need to ask are whether this monument precisely depicts balanced progression, whether the apex and the accumulation of materials in this formation reveals any kind of earliest ideology about the formation of the universe, and whether the degradation of the pyramid's base levels is indicative of the expansion of existence as we know it. To answer these questions we need to define the famous pyramids of Giza.

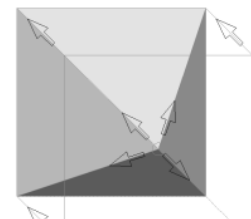

Figure 1. The progression from the Apex to the square base.

Several of the Giza pyramids are counted among the largest structures ever built (Watkin, 2005). The Egyptian sun god Ra, considered the father of all pharaohs, was said to have created himself from a pyramid-shaped mound of earth before creating all other gods. The pyramid's shape is thought to have symbolized the sun's rays (McCauley, 2012).

This study sheds light on the construction of this famous monument in Egypt, the pyramid of Cheops, also known as the great pyramid of Giza or the pyramid of Khufu. Simply because it is the only figure that has withstood the ravages of time. In addition, an undeniable truth about the nature of our universe lies within it like a secret mystery. Our research is based on the findings of the most recent international entrepreneurs, and aims to specifically expand the research on the pyramid of Giza.

On the other hand, the form of our expanding universe is described as closely related to the spreading out of the form of a pyramid, from its apex towards its base (Figure 1). To associate the above statements to this study, we have to look for patterns that incubate void within them.

What better and more precise starting point than both point and pointless beginning at the apex of the pyramid? The constancy of its structure sheds light on the norms by which the pyramids are constructed using pixels/blocks and void.

To first come up with a justification on the pyramid's formation, the first question that is imposed queries the nature of balance in the universe, although it is 
increasingly expanding using the norms of matter versus void. We might think that the universe is striving for balance throughout the chaos of possibilities. However, expansion acts in expandable orientation towards the undefined four corners of our universe, similar to the constitution of a pyramid.

Our persistence on the so-called theory of void and its role will be explained in detail in the findings, validating the previous statements. To start with, the Pyramid of Cheops has a link between the plans of each of its different levels/layers known as horizontal planes. The fundamental design of the whole structure is entwined with the balance of the pyramid itself. Throughout the different formations of the pyramid's horizontal blocks lies a mathematical explanation that accounts for the number of blocks in the horizontal alignment as well the constituents lying within.

The assumption is that figures that are symmetrical according to the horizontal and vertical axes as well as the diagonal axis all in all four directions are simple squares. These are similar to the formation created by five cubes linked to form a generic bigger square enclosure, which also conforms to the static state at the base level of any pyramid.

The configuration of four cubes linked to the four corners of the central cube starts from the top of the horizontal configuration of stones. Going down the inclination of pyramid, the set of multiple layers of blocks always adheres to the static alignment when viewed in the horizontal plane.

The continuous balance achieved in the alignment of the cubes is the secret to the planar configuration of the different pyramid levels (Figure 2).

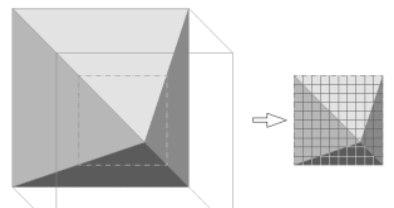

\section{Figure 2. Horizontal arrangement of Cheops pyramid.}

The Giza pyramid is always depicted in royal cubits (Rc). In the early 1880s, W.H.F. Petrie travelled to Giza; he was the first truly modern archaeologist to study the GP. His measurements on the Giza plateau in general, and on the GP in particular, are so accurate, they are still the main source of measurements used until today. Based on his measurements, it is now established that the exact length of an Egyptian royal cubit, Rc, as used for the GP, was $52.355 \mathrm{~cm}$ (Bryn, 2010). 


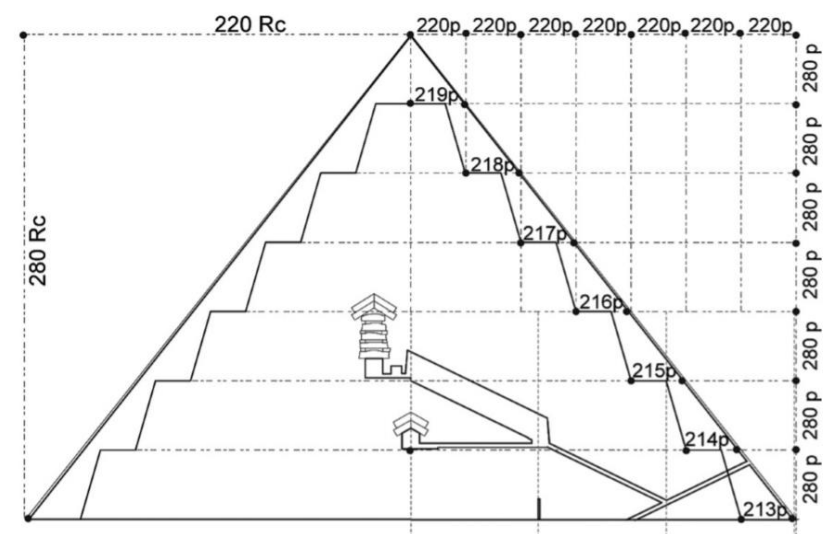

Figure 3. Pyramid section in royal cubits (Source: Bryn, 2010).

\section{Superimposition and interpolation}

In order to determine the void, and enhance understanding of its underlying states, there is a reliance on the following suppositions: The study uses two-dimensional compositions, as they are the simplest recognizable figures in the field of graphical understanding. Matter is represented as a filled geometrical block/pixel, while void is visualized as an empty square/pixel.

From another perspective, matter, which is in a state of motion, interchanges with energy, which can also assume a static condition. Quantified, the assumption is that the static state of elements will constitute a symmetrical representation in at least in four directions (X-axis, Y-axis, and two diagonals) in the orthogonal system of representation.

On the other hand, any state that does not constitute four-directional symmetry is definitely in a state of motion. The question that arises in this case is whether the empty space cubicle is static or dynamic. In this situation, expandable figures and their states are calculated in the different schemes, since single-symmetry or sole alignment is considered to be in movement, while four-symmetry, or the four-dimensional state is considered static.

The above interpretation of static and non-static states is basically derived from the construction of the Pyramid of Cheops, where it has been proven over time that its square base (symmetrical in four directions) is an indication of its structural stability and is in accordance with modern structural language.

The figures below will expand the study, offering a chance to attain results that will enable us to determine the notion of void and its characteristics. To start with the graphical presentation of the results, a representation is obtained from the configuration of equal and stable alignments of the different placements of cubes, and their underlying progressions. 
Figure 4 shows both the symmetrical configurations and non-symmetrical indications. What is about to be shown in figure 5 below is a matter deconstructed from combined cohesive states into individual states, under different conditions.

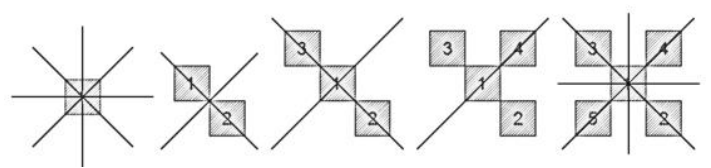

Figure 4. The representation of different arrangements for graphical allocation of pixels, where 1 cube and 5 cubes can achieve four-dimensional symmetry and are considered as being static or in a state of balance.

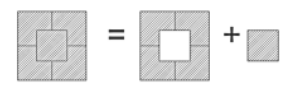

(a) State 1

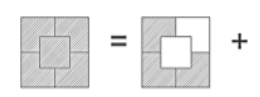

(b) State 2

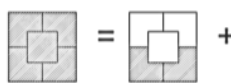

(c) State 3

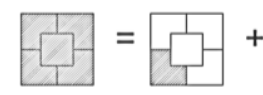

(d) State 4

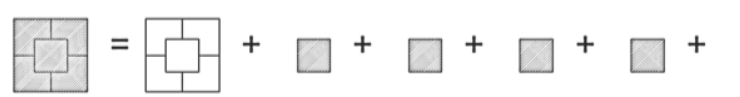

(e) State 5

Figure 5. Representation of graphical disintegration of pixels from cohesive states, from state 1 till state 5 .

Based on the cumulative values of States $1,2,3,4 \& 5$, the result obtained is as shown below in Figure 6. The figure below denotes the sequence of entities through progressive states.

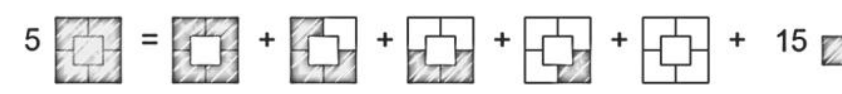

Figure 6. The cumulative of States 1 to 5. The left side equals the sum of the disintegrated states on the right side, $s^{\prime}$ ' $Y=25$ ' on the left side equals ' $X=25$ ' on the right side.

Applying this to the pyramid, apparently the problem is "the trouble at the top" (Lehner, 1997), viz. how the last blocks of masonry were laid near the apex point" (Bryn, 2010).

By using the geometric adjacencies of the paramount apex, with its four-sided pyramid at the beginning, across the sequence of expandable states, it is denoted that this apex cannot be occupied totally by building blocks/cubes/pixels. Rather, it is 
occupied by an initial pyramid having a base of nine square constituents and whose projection across the aperture/surface, in this case Cheops configuration, is calculated to a total of fifteen square areas designating fifteen negligible pixel states. Hence the calculation of the five states in Figure 6 will necessitate de-scoping the pyramid configuration, in other words, de-scoping 15 pixels/squares (Figure 7).

$$
15 \square=\text { Zero }
$$

Figure 7. Eliminating the 15 disintegrated states from the right side.

Below is an enhanced geometrical explanation (Figure 8).

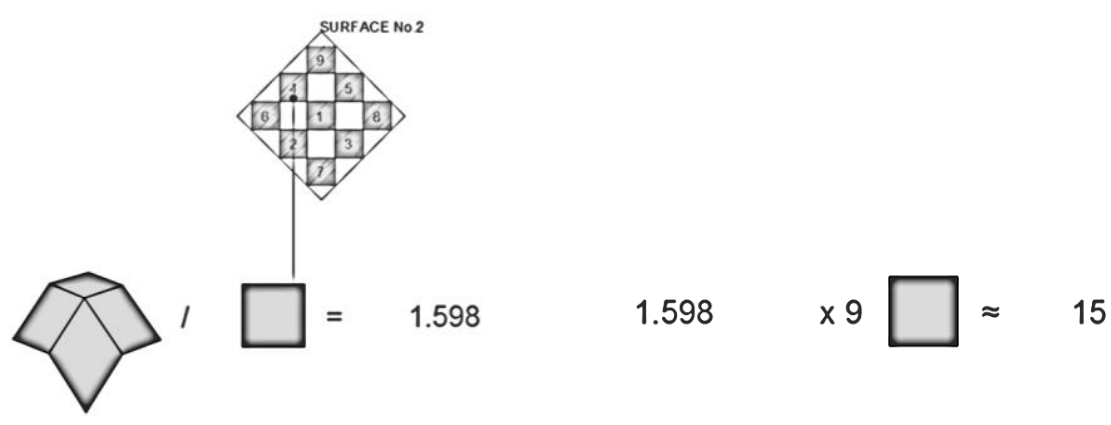

Figure 8. A pyramid has an area whose base, when projected over the surface of the Pyramid of Cheops, results in a surface area of 1.598 multiplied by the area of the square base.

The result of eliminating 15 cubes from the calculation is depicted in Figure 9.

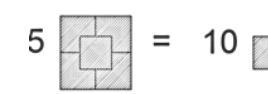

Figure 9. Sum of cohesive entities (pixels) on the left denoted as ' $Y$ ', equals the sum of disintegrated entities (pixels) denoted as ' $X$ ', as $Y$ (left) $=25$ is equivalent to $X$

$$
\text { (right) }=\mathbf{1 0} \text {. }
$$

The configuration of $\mathrm{Y}=25$ as seen in Figure 10 is symmetrical horizontally and vertically, as well as diagonally, which is equivalent to configuration of $\mathrm{X}=10$ (right side of the figure).

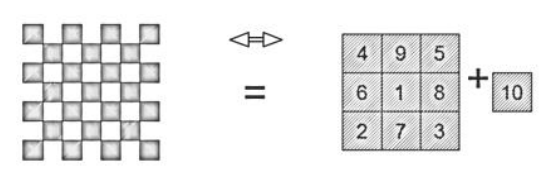

Figure 10. First data obtained is a coordinate of 10, 25.

According to the calculations, $\mathrm{Y}=5^{*} \mathrm{Y} 1=5^{*} 5$ is equivalent to $\mathrm{X}=5^{*} \mathrm{X} 1=5^{*}(\mathrm{X} 2+1)$. Second data obtained is a coordinate of $(\mathrm{X} 1=2, \mathrm{Y} 1=5)$, where 5 
cubes $(\mathrm{Y} 1=5)$ are static. Hence eliminating one figure from both ends will result in $\mathrm{Y} 1=\mathrm{Y} 2+1$. Then $\mathrm{Y} 2=4$ will be equivalent to $\mathrm{X} 2=1$, but a configuration of four cubes is non-static, hence $(1,4)$ is not the apex but rather the next configuration of $(2,5)$ denoting the least configuration of cubes to determine a solid state (static).

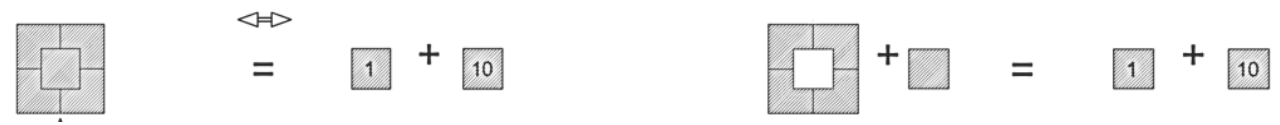

Figure 11. Denotes point $(2,5)$, apex point.

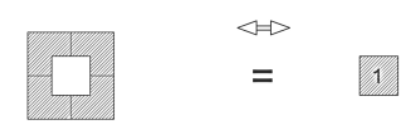

Figure 12. Denotes point $(1,4)$.

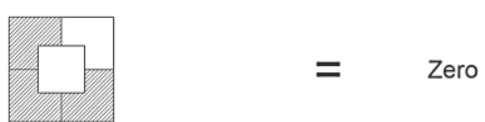

Figure 13. Denotes point $(0,3)$.

Propagating the findings of $Y=4$, according to $X=1$ as the bounding relation, the below superimpositions are found of good use, as the figure on the left is always symmetrical horizontally, vertically and diagonally when accumulating the cubes on the right. Accordingly, whenever four cubes are added to "Y" (which constitute symmetry when distributed equally to the four corners of any single square in the middle) there will be an addition of one square to the ' $\mathrm{X}$ ', as the simplest derived point is $(1,4)$. What will follow is an accumulation of four squares to ' $\mathrm{Y}$ ', and in turn, the addition of one square to ' $\mathrm{X}$ '.
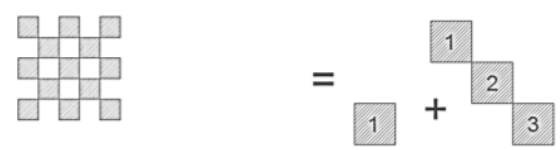

Figure 14. Point $(4,13)$.

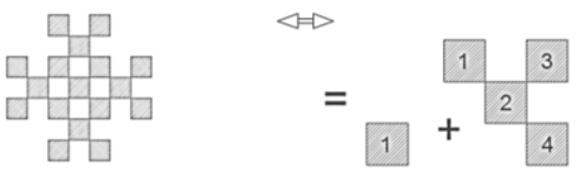

Figure 15. Point $(5,17)$.
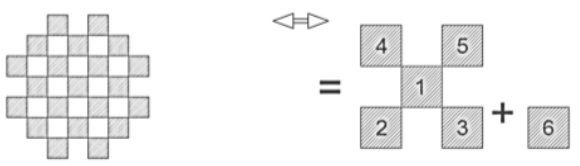

Figure 16. Point $(6,21)$. 

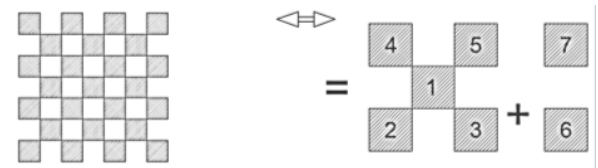

Figure 17. Point (7, 25).
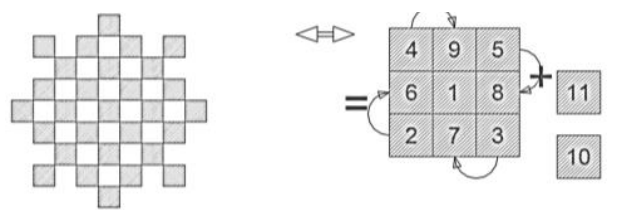

Figure 18. Point $(7,25)$.
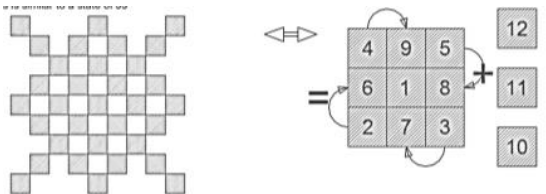

Figure 19. Point $(12,33)$.

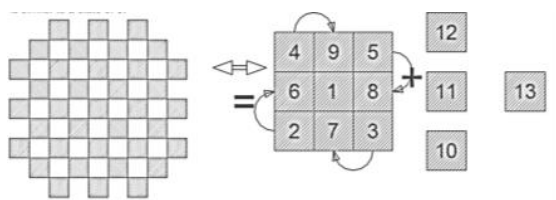

Figure 20. Points $(13,37)$ and $(14,41)$.

Whenever the apex $(2,5)$ is misplaced to become the origin $(0,0)$, the whole matter of static states in Figures 10 till 20 will be jeopardized to become non-static. Hence it is advisable for the apex to be located in its original location with coordinates of $(\mathrm{x}=2, \mathrm{y}=5)$.

\section{Superimposition of above results on the Pyramid of Cheops in Giza}

Many superimpositions exist when overlaying the section of the Giza pyramid (Cheops) with the graphical representations resulting from the different coordinate points derived from Figures 10 to 20.

Figure 21 demonstrates the first superimposition; the alignment between the graph of Cheops' section (having an angle at the apex of $76^{\circ}$ ). When cast upon the graph from the different coordinates obtained from the balanced figures, there is a match when superimposed over each other. The second superimposition is the match between the location of the opening/shaft on the face of the pyramid section and the Figure 17 state of coordinate $(6,21)$. The third superimposition is the length of the polyline resulting from different coordinates (from $(7,25)$ to $(14,41))$. Comparing its length using the graph, with the length of the polyline results in drawing the length of the tunnel inside the pyramid. Fourth, a superimposition is also obtained when 
calculating the length of distance between coordinates (from $(2,5)$ to $(14,41)$ in red and green combined) as equal to the length of the polyline taken from the corner of the pyramid added to the perpendicular line to the second side of the triangle. This in turn coincides with the horizontal alignment taken from the upper level of the upper King's chamber (5th and 6th superimposition).

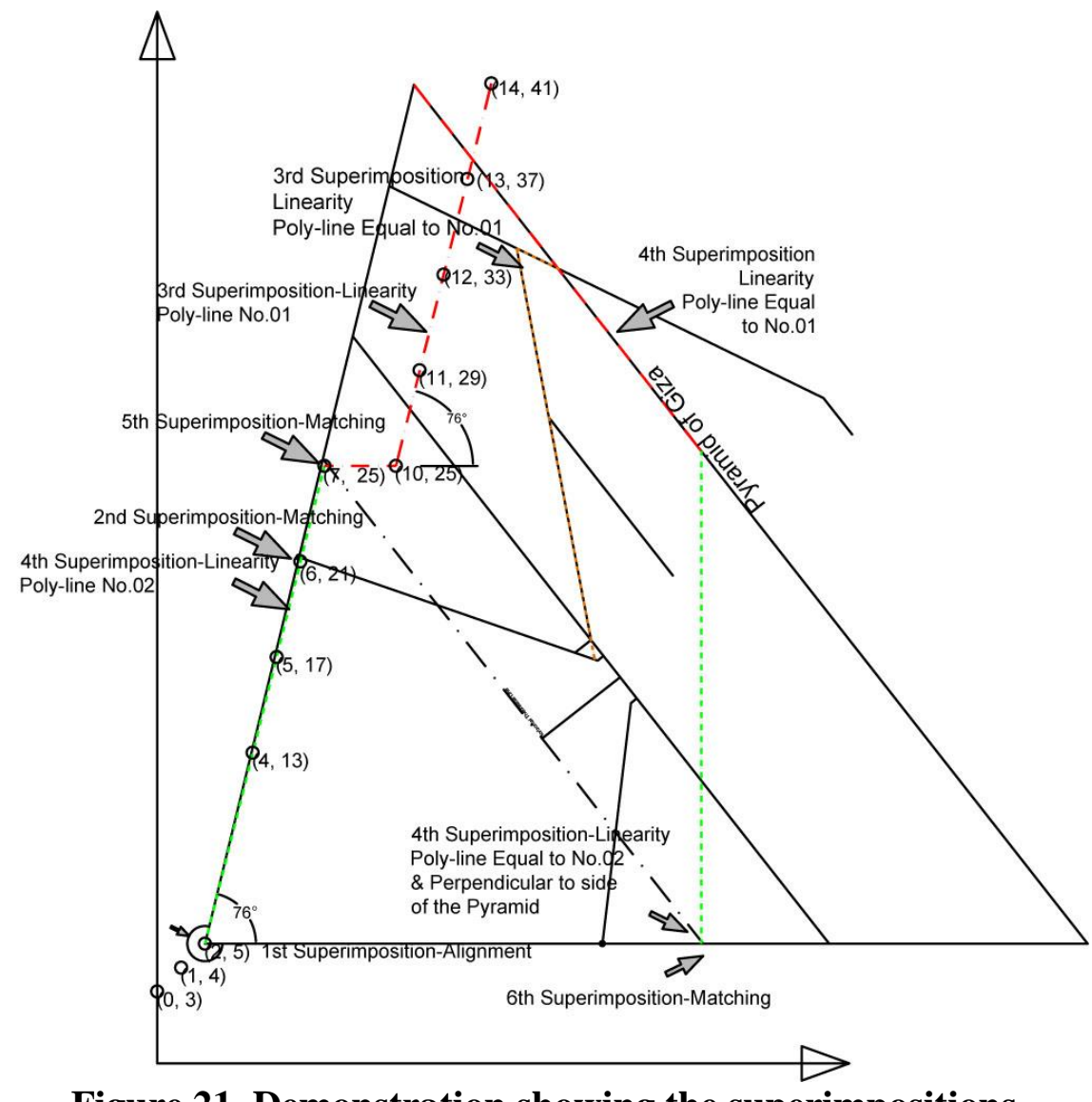

Figure 21. Demonstration showing the superimpositions.

The superimposition is shown in blue (different coordinates of points depicted from Figures 10 to 20) and black (depiction for Cheops pyramid on a scale of 1:5 in Rc, having a slope of $\mathrm{Y}=4 \mathrm{X}-3$ ).

\section{CONCLUSIONS}

As a result of our study, the finding of balanced configuration of horizontal building blocks confirm the theory of the natural balance between matter and void. The latter is depicted in accordance to the geometric figure of Cheops pyramid in Egypt.

The representation is well defined in the sequence of findings that resulted from simple justification for the Cheops Pyramid geometry that is derived from the patterns of its building blocks and their distribution from the apex downwards. 
This is a remarkable representation of the gradual derivative of balanced states in the depiction of Cheops pyramid. It sheds further light into the realms of mystery that has encompassed this remarkable landmark, and is of great concern to studies related to the prehistoric intelligence of a great civilization that used to inhabit the ancient world.

\section{REFERENCES}

Bryn, Ole Jørgen. 2010. Retracing Khufu's great pyramid the "diamond matrix" and the number 7. Nordic Journal of Architectural Research, 135-144.

Lehner, M. The Complete Pyramids: Solving the Ancient Mysteries. London: Thames \& Hudson; 1997.

McCauley, Marissa. 2012. A probing question: How were the Egyptian pyramids built? The Pennsylvania State University News.

Watkin, David. 2005. A History of Western Architecture (4th Ed.). Laurence King Publishing. p. 14. ISBN 978-1-85669-459-9."The Great Pyramid...is still one of the largest structures ever raised by man, its plan twice the size of St. Peter's in Rome". 